\title{
La campagne de glace de mon père avec Viktor Pokrovskij et ses cosaques
}

\author{
Alexandre BOURMEYSTER \\ Professeur émérite \\ Département de langues slaves \\ Université Stendhal - Grenoble 3 (FR) \\ abourmeyster@orange.fr
}

\section{Doi :10.5077/journals/connexe.2021.e609}

\begin{abstract}
Résumé
Lorsqu'on évoque la Campagne de glace [Ledjanoj pohod], on songe naturellement à l'Armée des volontaires partie de Rostov le 9 février 1918 sous le commandement du général Lavr Kornilov à la conquête d'Ekaterinodar. Toutefois, l'on ignore trop souvent le rôle décisif joué, à partir d’Ekaterinodar, par Victor Pokrovskij à la tête d’une armée du Kouban qui infligea de lourdes défaites aux bolcheviks dès janvier 1918.

Dans son récit, mon père, Nikolaj von Buhrmeister, officier de l'artillerie de la Garde, raconte comment il quitte le front et gagne Ekaterinodar à la fin du mois de décembre 1917, avec deux camarades de sa brigade. Ils s'engagent dans le corps de Volontaires que Viktor Pokrovskij forme sous l'égide de la Rada du Kouban. Avec leur artillerie, ils participent aux premières victoires remportées sur les bolcheviks, mais bientôt, le rapport des forces rend impossible la défense d'Ekaterinodar. Une fois les canons noyés, les artilleurs forment l'escorte de Pokrovskij, commandant de l'armée du Kouban, dans le repli qu'elle opère en montagne. À l'issue de combats incessants, elle effectue sa jonction avec l'armée des Volontaires de Kornilov. Après la mort de Kornilov et l'échec devant Ekaterinodar, la cavalerie de Pokrovskij assure la couverture de cette armée en retraite vers le Don et s'agrandit à mesure que s'accroît le recrutement des cosaques sous son impulsion. Mon père décrit avec vivacité les épisodes de cette épopée, insolite pour un ancien élève du Corps des Pages.
\end{abstract}

Mots-clés : Kouban, Ekaterinodar, cosaques, volontaires, bolchéviks.

\begin{abstract}
When one thinks of the Ice March [Ledjanoj pohod], one naturally thinks of the Volunteer Army that left Rostov on 9 February 1918 under the command of General Lavr Kornilov to conquer Ekaterinodar. However, the decisive role played from Ekaterinodar by Victor Pokrovskij at the head of the Kuban army, which inflicted heavy defeats on the Bolsheviks as early as January 1918, is too often ignored.

In his writings, my father, Nikolaj von Buhrmeister, an artillery officer of the Guard, tells how he left the front and reached Ekaterinodar at the end of December 1917, with two comrades from his brigade. They enlisted in the volunteer corps that Viktor Pokrovskij formed under the aegis of Kuban's Rada. With their artillery, they took part in the first victories over the Bolsheviks, but soon the balance of power made it impossible to defend Ekaterinodar. Once the cannons were drowned, the artillerymen formed the escort of Pokrovskij, commander of the Kuban army, in its retreat to the mountains. After constant fighting, it joined up with Kornilov's Volunteers Army. After Kornilov's death and the failure in front of Ekaterinodar, Pokrovskij's cavalry provided cover for this army retreating towards the Don and grew as the Cossack recruitment increased under his leadership. My father vividly describes the episodes of this epic, unusual for a former student of the Page Corps.
\end{abstract}

Keywords: Kuban, Ekaterinodar, Cossaks, volunteers, Bolsheviks. 
Note de l'auteur : La revue Connexe me donne l'occasion de faire connaître un document inédit, la narration par mon père de sa participation à la Première Campagne du Kouban ${ }^{1}$.

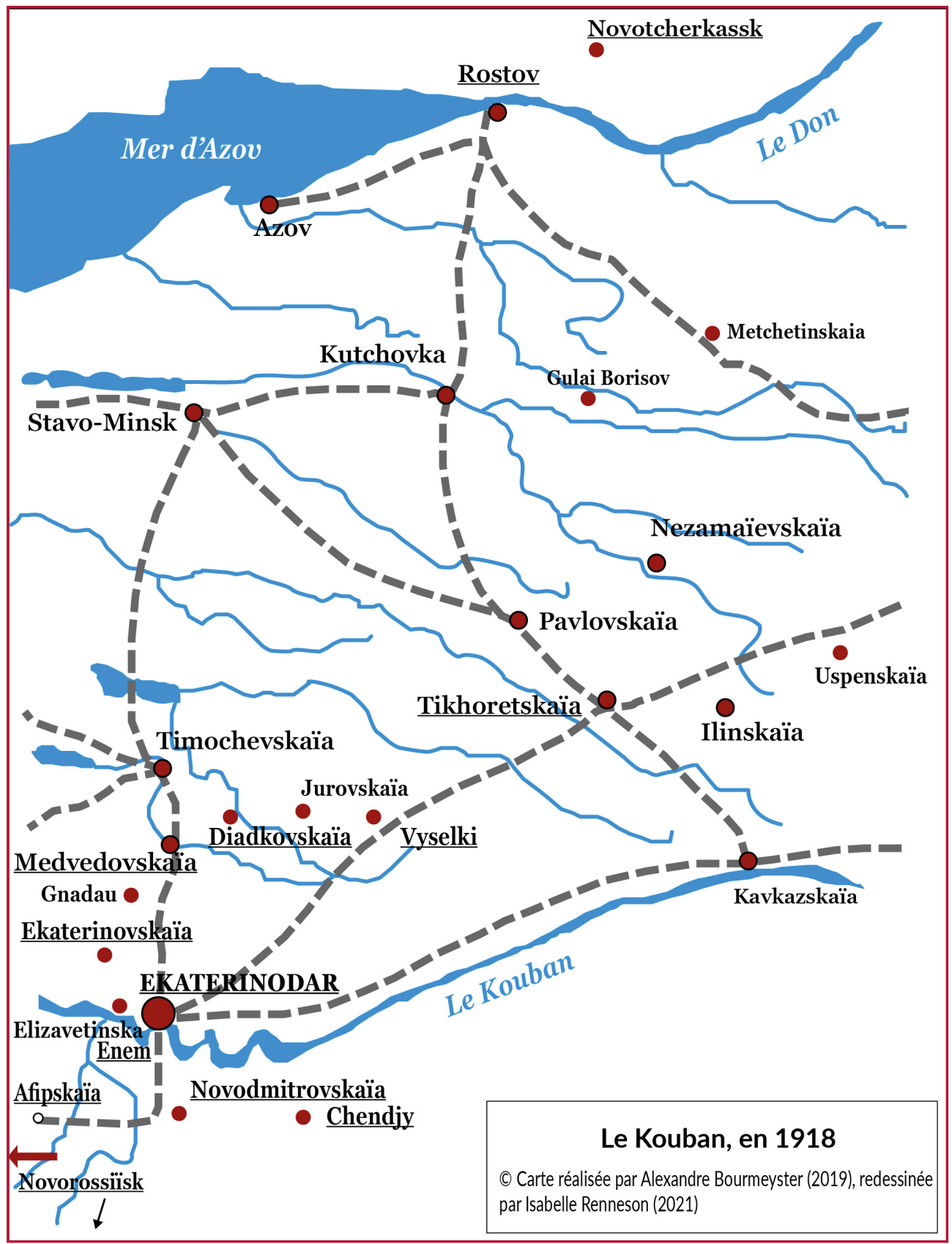

1 La majorité de la population du Kouban est d'origine cosaque, soit de la mer Noire, soit de la ligne du Caucase, installée sur le territoire des Tcherkesses depuis Catherine II. Après l'abdication de Nicolas II, en mars 1917, ils fondent une Rada militaire. Le 28 janvier 1918 est la date de proclamation d'une république populaire du Kouban. Le 16 février, Nikolaj Rjabovol devient chef du premier gouvernement. Le 1er mars 1918, les bolcheviks prennent Ekaterinodar. L’armée des volontaires reprend Ekaterinodar et rétablit la Rada. 
Lorsqu'on évoque cette épopée, baptisée Campagne de glace [Ledjanoj pohod], on songe naturellement à l'Armée des volontaires partie de Rostov le 9 février 1918 sous le commandement du général Lavr Kornilov (1870-1918 [voir Figure 1]) à la conquête d'Ekaterinodar (aujourd'hui Krasnodar), à sa mort devant Ekaterinodar (31 mars 1918), à sa relève par le général Anton Denikin (1872-1947 [voir Figure 2]) et à une retraite mouvementée qui permet de préserver l'Armée des volontaires pour une nouvelle campagne (23 juin-décembre 1918), victorieuse, celle-là. Mais on sousestime ou l'on ignore trop souvent le rôle décisif joué, à partir d'Ekaterinodar, par Viktor Pokrovskij (1889-1922 [voir Figure 3]) à la tête d'une armée du Kouban et infligeant de lourdes défaites aux bolcheviks dès janvier 1918.

En fondant cette Armée des volontaires [Dobrovol'českaja armija] le 15 novembre 1917 dans le sud de la Russie, à Novotcherkassk, la capitale des cosaques du Don, les généraux Mihail Alekseev (1857-1918 [voir Figure 4]), Kornilov et Denikin entendent, avec l'appui de l'ataman cosaque Aleksej Kaledin (1861-1918 [voir Figure 5]), utiliser la région du Don, un territoire riche et disposant de ses propres forces armées, afin de rassembler les forces hostiles au pouvoir soviétique. Les relations des généraux avec les cosaques du Don sont ambivalentes. S'il soutient et accueille les généraux, Kaledin, le premier ataman élu depuis 1708, n'aspire pas à s'engager avec eux dans la reconquête de la Russie, mais souhaite plutôt les voir partir au plus vite. Jouissant de divers droits et prérogatives, les cosaques sont surtout soucieux de préserver leurs terres et leur indépendance. L'appui des cosaques du Don permet à l'Armée des volontaires de reprendre Rostov-sur-le Don en décembre 1917, mais ils doivent l'abandonner à nouveau aux bolcheviks en février 1918. Les cosaques refusent de quitter le Don et Kaledin se suicide, alors que l'Armée des volontaires, sous le commandement du général Kornilov, prend la décision d'aller s'emparer d'Ekaterinodar. À travers les montagnes du Kouban, harcelée par les rouges, elle compte près de quatre mille combattants, dont plus de la moitié d'officiers, accompagnés d'un convoi de femmes et d'enfants. Pour Kornilov, Ekaterinodar sera le point de départ pour de nouvelles opérations contre les bolcheviks.

Or, depuis décembre 1917, c'est à Ekaterinodar même, que mandaté par la Rada, l'autorité politique des cosaques du Kouban, le capitaine Viktor Pokrovskij, héros de l'aviation militaire tsariste, entreprend de recruter des volontaires pour assurer la défense de la capitale du Kouban contre les bolcheviks. C'est alors que la vie de mon père croise le destin tumultueux du futur général Pokrovskij. 
Né en 1895, mon père, Nikolaj von Buhrmeister appartient à une lignée de seigneurs germano-baltes, de généraux, au service de la Russie des tsars depuis Pierre le Grand. Son père Johannes von Buhrmeister (1867-1898), lieutenant de la Garde, est mort prématurément phtisique. Mon père a été élevé par sa mère Marija Nevragina à Saint-Pétersbourg. Élève à l'École Pavlovsk puis au Corps des Pages, il est parti au front en décembre 1914 et a servi dans la première brigade d’Artillerie de la Garde, sans interruption, jusqu'à la révolution bolchevique. Avec le grade de capitaine, il part au Kouban combattre les bolcheviks.

\section{Nicolaj von BUHRMEISTER (1895-1976)}

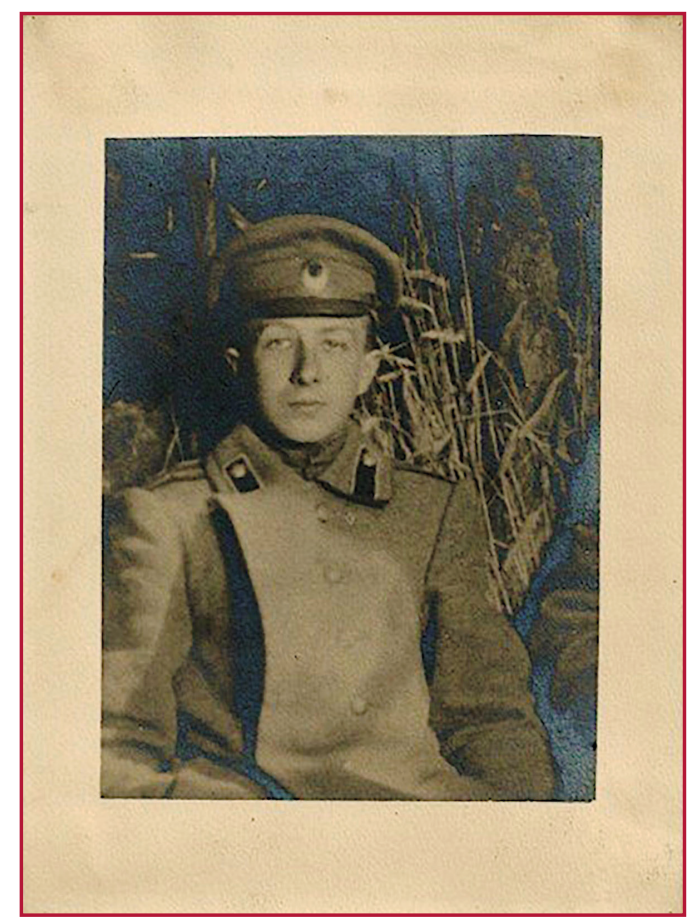

Sur le front en 1915 (auteur inconnu)
Armoiries Buhrmeister

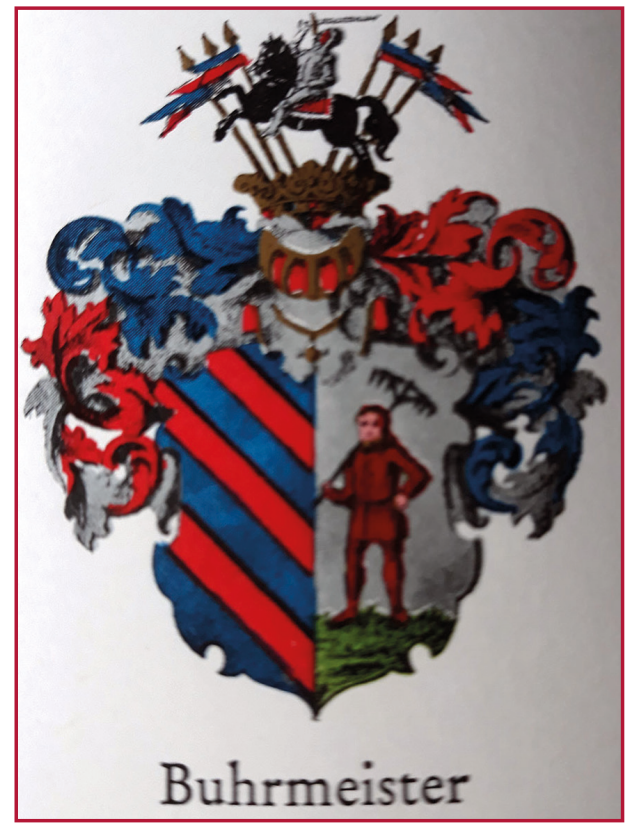

Source :

Patrick von Glasenapp. 1980. Baltisches Wappenbuch/Armorial balte/Baltic Armorial (p. 34). Alling (Allemagne) : Glasenapp Verlag. 
Alexandre BOURMEYSTER - La campagne de glace de mon père avec Viktor Pokrovskij et ses cosaques

C'est là que débute la narration de mon père. Il quitte Kiev le 24 décembre 1917 avec deux camarades de sa brigade, Nikitine et Matusevič, dontil ne se séparera guère durant toute la campagne. Revêtus de vieilles capotes sans épaulettes, coiffés de casquettes sans cocarde, dissimulant au mieux leur sabre et leur Nagan ${ }^{2}$, ils traversent en train une Ukraine déboussolée, contrôlée par des Gaïdamaks ${ }^{3}$, des bolcheviks, des prolétaires et enfin par des cosaques de "l'ancienne armée » qui leur demandent de retourner en première classe et leur permettent de reprendre une apparence "chrétienne ». Ils ne s'attardent pas à Rostov et préfèrent aller directement à Ekaterinodar, où, aux dires de Matusevič, s'organise la résistance aux rouges. La capitale du Kouban est surprenante par l'abondance des victuailles qui depuis longtemps sont devenues des raretés au nord. Bien que gouvernée par une Rada cosaque, elle est agitée par des manifestations bolcheviques. Le capitaine Pokrovskij, un ancien pilote militaire, connu pour ses exploits, est chargé par la Rada de former une troupe destinée à assurer son autorité. Pokrovskij recrute des volontaires parmi les officiers, les junkers et les cadets. Il est transporté de joie à la venue des trois artilleurs, qui plus est, des officiers de la Garde ! Il leur confie aussitôt le commandement de son artillerie ! Pour l'instant, elle n'existe pas. Ničego! Par une belle nuit, vingt-cinq artilleurs désarment, sans qu'ils s'y opposent, les cinq-cents plastuny ${ }^{4}$ d'un septième bataillon peu combattif et s'emparent, faute de canons, de leurs fusils et leurs mitrailleuses. Ils effectuent alors une mission qui a peu à voir avec leur vocation première : ils mettent fin à l'autorité du soviet d'Ekaterinodar qui siège (de façon subversive !) sous l'écriteau "Contrôle des documents de la ville d'Ekaterinodar ». Néanmoins, on a besoin de canons. Avec l'assentiment des autorités, nos artilleurs réquisitionnent les chevaux des fiacres et, par une nuit profonde, se rendent au jardin de la ville, dans le bâtiment réservé au théâtre d'été, où est conservé du matériel d'artillerie. Il est gardé par les plastuny. Nos artilleurs déjouent leur vigilance et récupèrent quatre canons datant de 1900. La batterie de la Garde est créée.

Le 21 janvier, on apprend que provenant de Novorossiïsk, près de 400 soldats démobilisés, des marins et des ouvriers portuaires avancent rapidement vers Ekaterinodar ; le soir même ils occupent Gueorgui-Afipskaïa, le dernier village avant la ville. Dans la chambre de Pokrovskij se tient un conseil militaire. Mon père y participe en tant qu'officier supérieur de la batterie. En tout et pour tout, Pokrovskij et le capitaine Galaev, organisateur lui aussi d'une formation de volontaires, disposent de 350-400 hommes, y compris les auxiliaires, de l'unité d'artillerie de la Garde,

2 Revolver adopté par l'armée russe depuis 1895. Il fut inventé par la firme belge Nagant.

3 Milice cosaque et paysanne contre la noblesse polonaise en Ukraine.

4 Fantassins cosaques dans l'armée russe au Kouban, chargés de la surveillance des frontières, des reconnaissances, à la manière des chasseurs, mobiles, invisibles ; tireurs d'élite armés de la vintovka, un fusil léger à canon rayé, un Stutzer, l'équivalent du riffle ou du mousqueton. Ils sont vêtus d'une tcherkesska.

CONNEXE 7| 2021 - La Guerre civile après 1917 : les échelles d'un conflit et sa représentation 
Alexandre BOURMEYSTER - La campagne de glace de mon père avec Viktor Pokrovskij et ses cosaques

(50 hommes), et de l'assurance du commandant de la cavalerie tcherkesse $\mathrm{e}^{5}$, le colonel Sultan Gerej (1880-1947 [voir Figure 6]), que les Tcherkesses leur sont favorables. À l'aube, les volontaires occupent le pont sur le Kouban à proximité d'Enem ${ }^{6}$, la première station ferroviaire en direction de Novorossiïsk. Le plan d'action de Pokrovskij est simple: Galaev avec toutes les mitrailleuses, les quatre canons et une centaine de fantassins occupera le pont et se manifestera de façon démonstrative. Pendant ce temps, Pokrovskij avec le reste des forces effectuera un profond détour et ira frapper au flanc les positions de l'ennemi.

$\mathrm{Au}$ matin, les bolcheviks en masse avancent vers le pont sur un étroit passage, le long de la voie ferrée. Le Kouban en crue a inondé les champs voisins. Les canons serrés roue contre roue, en travers de la voie et les mitrailleuses entrent en action. La foule des attaquants est si compacte et la distance si courte que la mise au point de la visée ne prend guère de temps. Les bolcheviks attaquent à plusieurs reprises sans succès. Au dernier assaut, particulièrement violent, ils prennent pied sur le pont. Ganaev avec une poignée de braves se lance dans une contre-attaque, et est tué. Tout semble perdu, mais les bolcheviks cèdent et s'enfuient en désordre car derrière eux crépite la fusillade. Pokrovskij est arrivé à temps ! Lorsque, "sur les épaules des fuyards », les artilleurs arrivent à Enem, tout est déjà fini. Sur le quai gisent des morts, avec parmi eux leur commandant, le ministre de la république autonome de Novorossiïsk, Jakovlev. Les bolcheviks ont fui vers Afipskaïa, abandonnant tout leur équipement, constitué de deux canons, deux obusiers et d'un stock d'obus.

Dans la nuit du 27 janvier, une attaque subite permet d'occuper Afipskaïa, les bolcheviks s'accrochent à la station, les artilleurs les dispersent. Mon père place sur la voie l'un de ses canons et par un tir direct fait exploser la chaudière d'une locomotive qui s'avance avec des renforts. " Afipskaïa est à nous ! ». Peu après arrive d'Ekaterinodar une députation de la Rada avec des félicitations à Pokrovskij et sa promotion au grade de colonel.

Début février, nouvelle alerte ! Vyselki est occupé par les soldats démobilisés d'un régiment d'infanterie du front caucasien. Les artilleurs débarquent du convoi à l'aube et suivent les fantassins déployés des deux côtés de la voie. Un vent violent déclenche la metel', la tempête de neige, les capotes trempées se transforment en glace. Les hommes se déplacent dans l'inconnu, aveuglés par le brouillard.

5 Circassiens, Adyguéens peuplant le nord-ouest du Caucase et confrontés pendant des siècles, sans unité politique, aux Huns, Avars, Khazars, Ottomans et Russes. Leur territoire est peu à peu cédé aux cosaques, utilisés en tant que troupes de couverture aux frontières de l'Empire russe durant la " pacification " du Caucase. Après la reddition de Chamil, en 1859, une vague d'exil des Tcherkesses a lieu vers l'Empire ottoman.

6 Nom d'un aoul tcherkesse célèbre dans l'histoire du Kouban en raison des querelles entre princes adyguéens et des conflits nés de la conquête du Caucase par la Russie. Il a été donné à une bourgade rattachée à la première station de chemin de fer sur la ligne Ekaterinodar-Novorossiïsk.

CONNEXE 7 | 2021 - La Guerre civile après 1917 : les échelles d'un conflit et sa représentation 
Bientôt éclate une violente fusillade :

À proximité de nos fantassins, je distingue les lignes des bolcheviks et j'ouvre le feu. Si nos efforts pour repousser l'adversaire n'aboutissent pas, ses attaques contre nous ne réussissent pas davantage. Dans la journée la chute de neige s'accentue, un vent glacé affronte nos visages, aucune visibilité. Nos lignes, comme celles de notre adversaire entretiennent, sans se voir, un combat inutile. À la tombée de la nuit, tout se calme. Pokrovskij laisse un cordon de sécurité et replie tout le monde à l'arrière, pour permettre à chacun de se réchauffer et se sécher. Sage précaution, il m'est ordonné d'embarquer les canons, mais les hommes épuisés au-delà de leurs limites ne peuvent le faire. Un quart d'heure de pause, un verre d'alcool légèrement coupé d'eau et les canons se retrouvent sur le quai. Si la situation est dure pour nous, elle l'est autant pour les bolcheviks. Durant la nuit, nos éclaireurs constatent que notre adversaire a quitté la gare et le village en direction de Tikhoretskaïa. À l'aube, nous occupons Vyselki sans combat. Pokrovskij repart à Ekaterinodar mobiliser des officiers pour renforcer nos rangs décimés.

Pendant ce temps, les bolcheviks commencent à menacer Ekaterinodar de tous les côtés.

La cause de notre malheur, c'est l'arrivée brutale du printemps. Le Kouban en crue commence à s'assécher et donne aux bolcheviks la possibilité de progresser ailleurs que le long des étroites voies ferrées et de déployer des forces bien supérieures aux nôtres. L'anneau se referme autour de nous et nous perdons tout contact avec le monde extérieur.

Une série de combats d'arrière-garde retarde cette avance, avec un épisode douloureux pour mon père, la perte de l'un de ses canons près de Vyselki en raison de l’incompétence d'un général, un juriste sans expérience militaire. Le 13 mars, le conseil militaire décide d'évacuer la ville. Les troupes, avec le minimum de bagages, doivent gagner les montagnes. La nuit, les artilleurs coulent leurs canons dans le Kouban, leur brigade est réduite à une centaine de cavaliers et devient l'escorte de Pokrovskij, le commandant en chef de l'armée du Kouban. À l'aube, cette armée de volontaires se met en route en direction de l'aoul ${ }^{7}$ tcherkesse Tikhtomoukhaï. En dehors de l'escorte (Nikitin à sa tête, Matusevič et mon père, ses adjoints), elle est composée du régiment de tirailleurs du colonel Tunenberg, (1200 hommes : 700 officiers, 400 junkers, 100 cadets, quatre mitrailleuses) d'une batterie de deux canons, de deux escadrons de cavalerie tcherkesse (500 hommes, quatre mitrailleuses), des escadrons du colonel Kuznecov, du colonel Demjanik, du général Sergej Ulagaj (1875-1944 [voir Figure 7]) qui regroupent chacun une centaine d'officiers, et enfin d'une milice destinée à protéger la caisse de l'armée.

Le plan de Pokrovskij est de trouver un asile sûr pour une résidence provisoire. Accueillis chaleureusement par les Tcherkesses, les volontaires ne restent pas longtemps à l'abri. Leur arrière-garde est exterminée, leur avant-garde se heurte à

7 Village du Caucase.

CONNEXE 7| 2021 - La Guerre civile après 1917 : les échelles d'un conflit et sa représentation 
Alexandre BOURMEYSTER - La campagne de glace de mon père avec Viktor Pokrovskij et ses cosaques

« l'armée de Sorokin » (Ivan Sorokin 1884-1918 [voir Figure 8]) ; battue à Vyselki, elle se dirige vers Ekaterinodar, par une voie détournée. À la station Penzenskaïa, on apprend que Kornilov a quitté Rostov et progresse dans le Kouban. La cruelle randonnée à travers les montagnes s'achève par la jonction avec les troupes exténuées de Kornilov le 9 avril. Kornilov exige de Pokrovskij qu'il lui cède le commandement de ses troupes, Pokrovskij s'obstine à vouloir conserver l'autonomie de « l'armée du Kouban » placée sous l'égide de la Rada. Le 14 mars, Kornilov entreprend depuis Chendjy l'attaque de Novodmitrovskaïa. Alors que durant trois jours et trois nuits sans interruption sévit la tempête de neige, ses troupes doivent traverser à gué, ou à la nage, une modeste rivière de montagne au courant très rapide, dont les anciens passages ont été inondés ou bien détruits par les rouges. Cette traversée sera baptisée campagne de glace. Les rouges se défendent opiniâtrement.

Pokrovskij a ététraité de haut, ses troupes n'interviennent qu'à la fin dela deuxième journée, après d'intenses négociations. Il s'est résigné : ses fantassins passent sous le commandement du général Sergej Markov (1878-1918 [voir Figure 9]), ses cavaliers sous celui du général Ivan Erdely (1870-1939 [voir Figure 10]). Reste sous son autorité son escorte, et une tâche, celle de mobiliser sous son autorité les cosaques du Kouban dans de nouvelles formations.

Pokrovskij confie à mon père diverses missions. Mon père les relate, notamment ses entrevues avec les généraux Kornilov, Markov, Afrikan Bogaevskij (1872-1934 [voir Figure 11]), Erdely, et surtout celle où il fait la connaissance du général Denikin, le successeur de Kornilov. Sur la route vers Medvedovskaïa, une compagnie de génie restaure un vieux pont à moitié en ruine. Pokrovskij reçoit l'ordre d'installer une unité pour contrôler le passage et confie cette tâche à mon père :

J'ai déjà réussi à contrecarrer quelques tentatives lorsque je vois, se dirigeant directement vers moi, un monsieur coiffé d'un bonnet de fourrure et vêtu d'un bekech civil sans épaulettes avec un col d'astrakan. Ainsi s'habillent les membres du Conseil régional et ceux de la Rada, By, Riabovol, et d'autres, des carriéristes, des politiciens, que nous n’apprécions guère. Voyant la ferme intention de l'intéressé d'aller jusqu'au pont, je l'informe du but de ma présence ici. Aucune réaction. Je répète d'une façon plus vive. Le même résultat. Alors je m’adresse à lui de façon catégorique, et mon interlocuteur, hors de lui, ouvre son bekech, sous lequel je découvre une tunique avec des aiguillettes d'état-major et Saint-Georges au cou : «Vous êtes fou! Je suis le commandant en chef de l'Armée des Volontaires, le général Denikin! Je vais vous faire fusiller ! ». Je lui rends les honneurs et lui cède le passage.

Autre mission : la mobilisation à Ekaterinovskaïa est interrompue par l'approche des rouges. Pokrovskij confie à mon père le commandement des plastuny, lui-même part stopper l'avance de l'adversaire avec les cosaques qui ont des chevaux :

Tous mes plastuny sont des vieux avec des barbes larges comme des pelles, ils 
sont armés de fusils Berdan ${ }^{8}$. Je les sors du village, je les dispose en ligne, selon toutes les règles de l'art et je pars en avant... Mes vieux avancent vaillamment et tout se passe bien jusqu'à ce que nous nous trouvions à mille pas de l'adversaire. Les bolcheviks ouvrent sur nous un feu nourri, mes barbus se couchent et il est impossible de les relever. Arrêter l'ennemi par le feu ? Chacun d'eux n'a que cinq cartouches !... Pokrovskij m'envoie l'ordre de me replier vers le village... jusqu'à son poste de commandement où il m'attendra. Je transmets cet ordre à mes hommes en leur recommandant de se replier un par un. À peine l'ai-je donné que je vois mes lignes se lever et tous comme des lapins déguerpissent vers le village et semblent disparaître sous terre. En quelques minutes, je me retrouve tout seul, « comme un doigt en plein champ ».

Contraint de suivre leur exemple, mon père retrouve son ordonnance Dubov avec leurs chevaux. Aucune trace de Pokrovskij, mais une foule de vieux et de vieilles qui crient à leur venue : "Canailles ! Vous nous mobilisez et maintenant les rouges vont nous écorcher vifs! ». Quelqu'un hurle : «À quoi bon discuter avec eux, tirez-les de leurs chevaux et battez-les!». Dubov frappe le cheval de mon père de violents coups de fouet, il se cabre, la foule s'écarte et laisse la place à mon père et à Dubov pour prendre la fuite, accompagnés de cris et d’injures. Poursuivis par des cavaliers rouges, ils ne rejoignent Pokrovskij et ses escadrons que tard le soir. La seule chose qu'il entend de sa part, c'est : « Et moi je pensais que vous ne vous en tireriez pas !». Tous ceux qui se souviennent de lui le confirmeront : il n'accordait qu'un très faible prix à la vie humaine... à la sienne entre autres.

Durant la retraite, l'Armée des volontaires et celle du Kouban suivent d'abord le même itinéraire. Une halte de deux jours à Diadkovskaïa permet, non seulement de se reposer, mais pour la première fois, depuis l'abandon d'Ekaterinodar, de se laver et de se débarrasser de ses poux.

Le matin du 5 avril, nous quittons Diadkovskaïa. Je n'oublierai jamais ce départ. Nous passons devant l'école, un lieu où, comme d'habitude, on installe l'hôpital. Nous partons vers l'inconnu, menacés de tous côtés. Le nombre des blessés est énorme, surtout après la bataille d'Ekaterinodar, il dépasse quasiment celui des soldats valides. L'armée s'est transformée en un interminable convoi sanitaire. Autant que ses blessés, elle est menacée de périr. La décision est prise de laisser à Diadkovskaïa, tous les grands blessés. Auprès d'eux restera une partie du personnel médical, des volontaires. Nous passons devant l'école. Aux portes, clopinent, se cramponnent, rampent des malheureux qui nous accompagnent de lamentations, de gémissements, de malédictions. Il semble que les bolcheviks se soient comportés humainement avec eux. Seules quelques personnes auraient été fusillées. Mesure cruelle, héroïque, mais incontestablement salutaire pour l'armée des volontaires, elle lui permet de retrouver sa mobilité. Dès le départ de Diadkovskaïa, l'itinéraire

8 Le fusil Berdan, créé par l'Américain Hiram Berdan et adopté par l'armée russe de 1870 à 1891, est une arme de calibre 11, 67, à un coup. Il équipe encore l’infanterie pendant la guerre de 1914-1917, bien qu’il soit remplacé à partir de 1891 par le Mosin-Nagant, calibre 7,62. 
de Pokrovskij va différer de celui l'Armée des volontaires, notre calendrier ne coïncidera plus toujours avec le leur. Nous sommes en perpétuel déplacement. La mission confiée à Pokrovskij est de mobiliser des cavaliers pour l'armée du Kouban et des plastuny pour compléter les effectifs de l'infanterie. Nous partons pour des raids de deux, trois jours, effectuons des boucles, tournons dans des cercles et rejoignons le gros des troupes à l'avant-garde ou à l'arrière-garde. Cette tactique se révèle doublement utile, elle permet de verser dans l'armée des forces nouvelles, et de la protéger avec un rideau d'unités de cavalerie constamment en mouvement qui égarent par leur mobilité les bolcheviks. Au bout de deux semaines, Pokrovskij dispose déjà d'une division de cavalerie avec d'excellents chevaux car les cosaques viennent nombreux, à la fois montés et armés.

Le récit de mon père comporte des scènes spectaculaires, comme, lorsque masquant leur infériorité numérique par les nuages de poussière de la steppe, Pokrovskij déploie en une lave unique, sur un large front, le long des collines, ses 500-600 cosaques qui sabrent vigoureusement, sèment la panique parmi les rouges et bousculent leurs taganki $i^{9}$. Mais aussi des moments émouvants, comme le 27 avril, la nuit pascale :

Nous sommes rangés par six, à cheval, attendant, sans nous presser, qu'on nous ordonne d'aller poursuivre l'ennemi, une fois qu'il sera battu. À gauche, dans une combe, l'église très éclairée. Passe la procession de la Croix, quelques échos des chants nous parviennent: "le Christ est ressuscité !». Je ne peux oublier le contraste qui me saisit : une nuit d'amour et de pardon universel, une nuit claire, et nous qui attendons de nous ruer à nouveau pour tuer nos semblables.

Ou encore l'éloge de la générosité cosaque :

Avec quelle gentillesse nous sommes accueillis! Je commence littéralement à étouffer sous les friandises pascales, les mets succulents et les boissons. Chez nous, au Nord, il en est autrement. Ici pour la première fois, je découvre la réalité d'une incroyable abondance. Les cosaques sont particulièrement hospitaliers ; tu marches dans la rue, on te sollicite : viens manger du kuličc ${ }^{10}$, viens boire un verre de vodka... Je me souviens d'une vieille qui m'a fait rire. Nous sommes vêtus d'une tcherkeska ${ }^{11}$ et ne nous distinguons guère des cosaques ; elle me demande : "Et toi, kasatik (mon petit), de quelle stanica ${ }^{12}$ tu viens ? » Je lui réponds : « de Saint-Pétersbourg ». «C'est dans quel district? C'est loin? »

9 Véhicule avec une suspension à ressorts, du même type que le fiacre, attelé à deux ou parfois à trois ou quatre chevaux, avec une mitrailleuse (une Maxima) sur son affût pointé dans le sens contraire à la direction. Il transporte trois hommes : le cocher, le mitrailleur et son adjoint, et peut effectuer un tir pointé tout en roulant. Léger, manœuvrable, il peut accompagner les raids et les attaques de la cavalerie. Il peut aussi servir à transporter des fantassins à la vitesse d'un cheval au trot. Son grand utilisateur sinon son inventeur fut Nestor Makhno dans ses équipées à travers l'Ukraine.

10 Brioche, gâteau traditionnel de Pâques.

11 Manteau traditionnel caucasien pour homme, de couleur noire, descendant jusqu'aux genoux, avec de larges manches, cintré à la taille, orné sur la poitrine de " cartouchières ", destinées initialement à contenir de la poudre. Il s'agit de l'uniforme des cosaques du Kouban et du Terek, et aussi, de la tenue adoptée dans les guerres ou les cérémonies, par des hauts gradés, notamment par le général Wrangel.

12 Village de Cosaques.

CONNEXE 7 | 2021 - La Guerre civile après 1917 : les échelles d'un conflit et sa représentation 
Après cette première campagne du Kouban, mon père quitte Pokrovskij en juin 1918, mais ne rejoint pas l'Armée des volontaires de Denikin dans la seconde campagne, sans doute pour des raisons politiques : mon père est monarchiste. À Odessa, il participe à la mobilisation d'une armée blanche pour résister à l'avancée des rouges en Ukraine. Il est évacué sur Malte, lorsque les Anglais quittent Odessa (avril 1919). Il retourne au combat, et lorsqu'en avril 1920, Pëtr Wrangel (1878-1928 [voir Figure 12]) remplace Denikin à la tête d'une armée russe, il participe à toutes ses opérations, la bataille pour Kiev, le Kouban, la défense dela Crimée, jusqu'àl'évacuation de Sébastopol avec Wrangel, et le grade de colonel, en novembre $1920^{13}$. Évacué à Dubrovnik, il séjourne en Yougoslavie jusqu'en 1924, puis part en exil en France. Dans la banlieue lyonnaise, ouvrier dans une grande usine de textiles artificiels, il fait peu à peu une carrière de chimiste, épouse en 1929 Marija Evrejnova, la fille du dernier gouverneur de Penza en Russie. Ils ont deux fils : Alexandre 1930- ... et Ivan 1938-2004. En 1939, il se porte volontaire pour défendre la France et suit des cours de préparation militaire jusqu'à la débâcle de 1940. Il n'en oublie pas moins son devoir à l'égard de la Russie. Membre de l'Union des Anciens Combattants Russes, le ROVS, cet ennemi juré des bolcheviks créé par le général Wrangel, il dirige sa branche à Lyon de 1940 à 1948, durant la difficile période de l'Occupation. En outre, il anime de nombreuses associations d'émigrés par des publications, des conférences d'histoire, écrit une histoire de sa chère brigade d'artillerie publiée depuis en Russie. Convaincu que le régime soviétique sort renforcé de la guerre, il demande la nationalité française et l'obtient aussitôt. Son attachement à la France ne lui fait pas oublier, jusqu'à sa mort, un profond sentiment de solidarité envers ses frères d'armes dans le besoin. Sa devise : « Nous périssons tous, mais nous ne nous rendons pas ».

13 Les idées politiques de Denikin : il accepte, comme Kornilov, la révolution de Février ; il est partisan d'une monarchie constitutionnelle. Il s'en remet aux décisions de l'Assemblée constituante pour le régime futur. En attendant, il soutient l'idée de laisser la possession des terres à ceux qui la détiennent de facto et promeut une Russie grande, unie, indivisible. Il est favorable à la langue russe officielle avec l'ukrainien dans l'administration et l'enseignement. Les idées politiques de Wrangel : il désapprouve l'abdication du tsar, pour lui source de tous les conflits, du désordre dans l'armée et de l'absence d'une politique concrète. Opposé à la " marche sur Moscou ", il accorde la priorité à la jonction avec les forces de Kolčak à partir de Tsaritsyne. Il fait preuve de réalisme politique quand il reconnait la fédération du Nord-Caucase, les revendications ukrainiennes, Nestor Makhno, et l'autonomie des terres cosaques. 
Ce récit de la Campagne de glace avec Pokrovskij, je l'ai trouvé dans les archives de mon père , parmi bien d'autres documents. Il est rédigé au crayon au dos de feuilles millimétrées de son labo de chimie. Désireux, vingt ans après les événements qu'il décrit, de donner une place dans la tourmente de la guerre civile à sa chère brigade d'artillerie, créée par Pierre le Grand, il doit la réduire à une batterie à Ekaterinodar, puis à l'escorte du commandant en chef de l'armée cosaque du Kouban! Mais il éclaire d'une façon inédite la personnalité de Viktor Pokrovskij, trop souvent négligé ou dénigré par ses contemporains et les historiens.

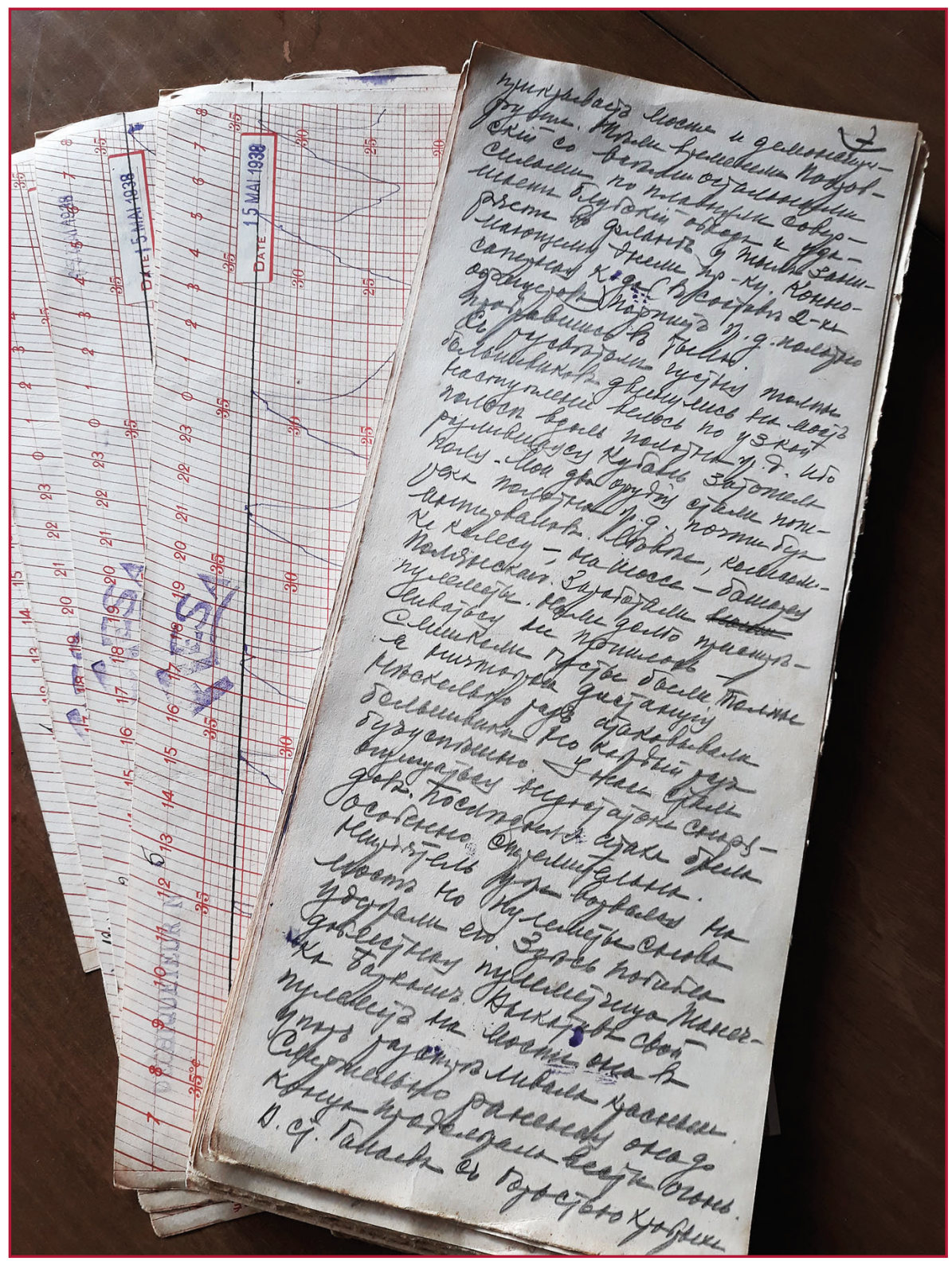

Extraits manuscrit (Archives 1938) 


\section{Personnalités mentionnées dans l'article}

(Toutes les images sont du Domaine public)

Figure 1. Lavr KORNILOV

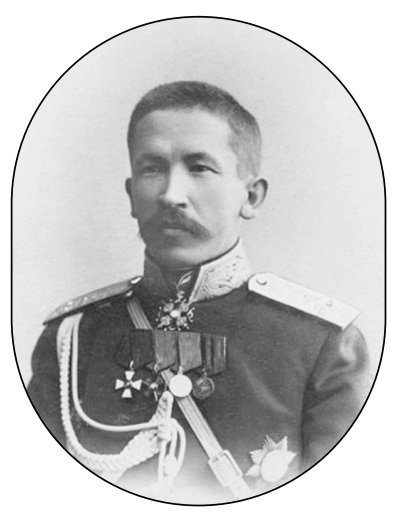

Figure 2. Anton DENIKIN

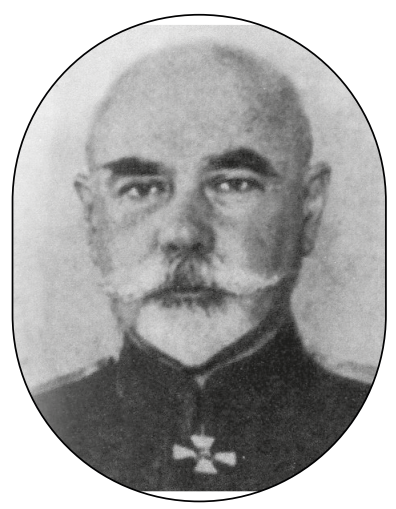

Cosaque originaire du Kazakhstan, Lavr Kornilov étudie à Omsk, effectue des missions d'exploration en Asie centrale, participe à la guerre russojaponaise, à Moukden, et est décoré de l’Ordre de Saint-Georges. En 1914, il est général-major dans la huitième armée d’Aleksej Brusilov. Capturé, évadé, promu général d'infanterie, il participe à l'offensive de Brusilov, commande la zone militaire de Petrograd en mars 1917, et crée un illustre régiment d’assaut. En juillet, succédant à Brusilov à la tête de l'armée russe, et avec l'accord apparent de Kerenskij, il cherche à rétablir l'ordre. À la suite de son intervention à Petrograd, présentée comme un coup d’État par Kerenskij, il est arrêté avec Denikin, Markov et Romanovskij. Retenus à Buhov, ils s'évadent après la révolution d'Octobre, gagnent le Don et rejoignent le général Alekseev. Kornilov prend le commandement militaire de l'armée des volontaires, qui entame en février 1918 la première campagne du Kouban avec pour objectif la libération d'Ekaterinodar tombée aux mains des bolcheviks. Au cours de l'assaut, Kornilov est tué le 13 avril 1918 par un éclat d'obus dans son quartier général. Il reste l'objet d'un culte chez les kornilovcy, les impitoyables combattants du régiment d'assaut de Kornilov, force primordiale de l'armée des volontaires.

En août 1914, Anton Denikin commande la région militaire de Kiev. En 1916, il est à la tête de la huitième armée et participe à l'offensive de Brusilov en Roumanie. Arrêté et enfermé à Bykhov avec Kornilov, il fuit avec lui pour former l'armée des volontaires à Novotcherkassk. Dans la première campagne du Kouban, il est le second de Kornilov, puis le remplace après sa mort, le 31 mars 1918. Après le ralliement des cosaques du Don, il dirige la seconde campagne du Kouban, fin juin 1918, qui aboutit à la prise d'Ekaterinodar. Le 8 janvier 1919 se produit la fusion de l'armée des volontaires et de l'armée du Don dans les Forces armées de la Russie du Sud. Le 24 juin, Denikin est nommé commandant en chef par le gouvernement d'Omsk. Le 3 juillet 1919, il annonce le début de la marche sur Moscou. Après une avance maximale jusqu'à Orel et Toula, la situation se dégrade en octobre. Durant l'hiver 1919-1920, Denikin doit évacuer l'Ukraine, le Kouban. Les 26-27 mars 1920 a lieu l'embarquement dramatique à Novorossiïsk vers la Crimée. Le 4 avril 1920, Denikin démissionne au profit de Wrangel et part pour l'Angleterre. Il s'exile en France à Mimizan. En 1933, il apporte un soutien public à l'Armée rouge : "face au pire ennemi de la Russie et du peuple russe, l'Allemagne nazie ». En 1945, il part pour les USA avec 2000 pages de Mémoires à publier. Il meurt en 1947 à Ann Arbor dans le Michigan. Ses restes sont transférés au monastère de Donskoï auprès de ceux d'Ivan Il'in, d'Ivan Šmelëv, du patriarche Tihon, le 3 octobre 2005. 


\section{Figure 3. Viktor POKROVSKIJ}

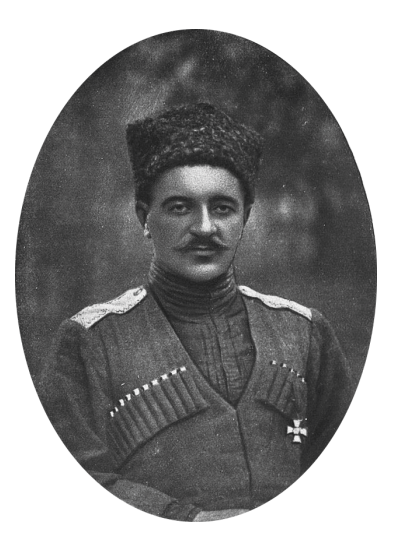

Issu d'une famille noble, Viktor Pokrovskij étudie à l'école militaire Pavlovsk, puis à l'école militaire d'aviation en 1914 à Sébastopol. Pilote observateur, il reçoit l'Ordre de Saint-Georges pour avoir forcé à atterrir un avion autrichien et avoir capturé ses pilotes en juin 1915. Il est promu capitaine en octobre 1916. Au Kouban, sur la demande de la Rada, il forme un corps de volontaires et inflige une cruelle défaite aux bolcheviks à Enem et Gueorgui-Afipskaïa, les 21-23 janvier 1918. Il doit évacuer Ekaterinodar le 13 mars sous la pression des troupes rouges commandées par Sorokin. Promu colonel par la Rada, puis général-major, commandant de l'armée du Kouban (3000 hommes), il rejoint le 27 mars l'armée des volontaires de Kornilov (2700 hommes, dont 700 blessés) et se place sous son commandement. Successivement commandant de la première brigade, de la première division de cavalerie, du premier corps d'armée du Kouban, il participe à la prise de Tsaritsyne, mais aussi à la «tragédie de Maïkop ", où ses cosaques massacrent en représailles 2000 prisonniers, les 21-22 septembre 1918. Sous les ordres de Wrangel, il dissout la Rada du Kouban accusée de séparatisme. Il le remplace au commandement de l'armée du Caucase. Battu par l’Armée rouge, il émigre en Bulgarie et prépare un débarquement au Kouban, dans la clandestinité. Il est tué par la police bulgare, lors d'une interpellation.

Figure 4. Mihail ALEKSEEV

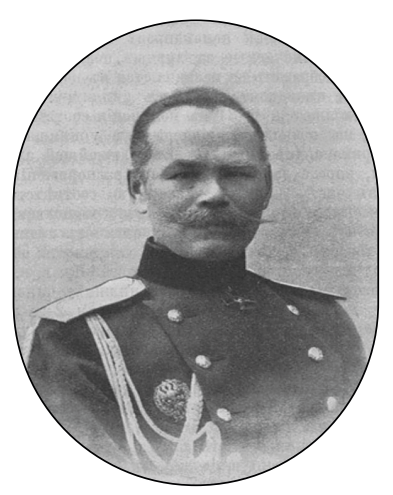

Mihail Alekseev participe à trois guerres : la guerre russo-turque (18771878), la guerre russo-japonaise en Mandchourie et la Grande guerre. En 1914, il est général d’armée, homme de confiance de Nicolas II. Il est promu en 1915, devient chef d'état-major à la Stavka, lorsque le tsar prend personnellement le commandement suprême. Ses relations avec Mihail Rodzjanko, président de la quatrième Douma entre 1912 et 1917, ainsi que sa responsabilité dans les événements qui aboutissent à l'abdication du tsar, restent objets de désaccords entre les historiens. Sa préoccupation principale aurait été la préparation de l'offensive programmée pour avril 1917 en commun avec les Alliés. Toujours à la tête de la Stavka, il doit ordonner l'arrestation de Kornilov et de ses proches après les événements d'août 1917. Il démissionne par la suite. Après le coup d'État bolchevique, il gagne Novotcherkassk, rejoint par Kornilov. Il organise les structures de l'armée des volontaires, participe aux deux campagnes du Kouban en tant que responsable administratif et financier. Il assiste à la conférence politique d'Oufim en septembre 1918 et meurt de congestion pulmonaire le 8 octobre de la même année.

Figure 5. Aleksej KALEDIN

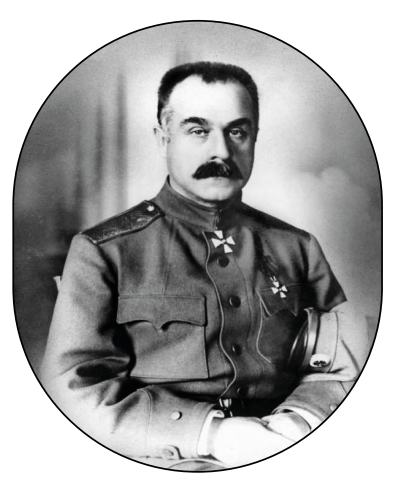

Né à Novotcherkassk, Aleksej Kaledin va à l'école militaire, à l'académie d'état-major. Pendant la guerre, il commande la douzième division de cavalerie, puis la huitième armée sur le front sud-ouest, après Brusilov. Relevé de ses fonctions par le gouvernement provisoire, il est élu ataman des cosaques du Don et dirige le gouvernement militaire. Partisan de Kornilov, il l'accueille à Novotcherkassk avec Alekseev, mais ce n'est qu'après la prise par les bolcheviks de Taganrog et Rostov qu'il s'engage à coopérer à la formation de l'armée des volontaires. Faute de cosaques prêts à le suivre, il démissionne et se suicide le 30 janvier 1918. 
Figure 6. Sultan GEREJ

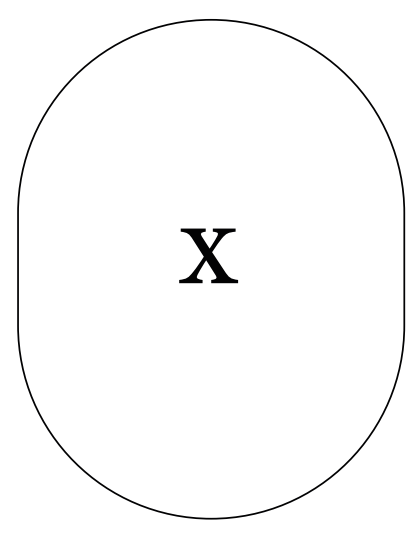

Figure 7. Sergej ULAGAJ

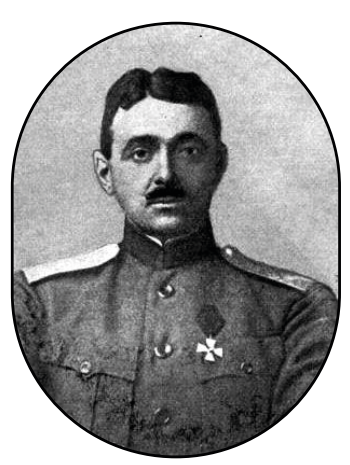

Figure 8. Ivan SOROKIN

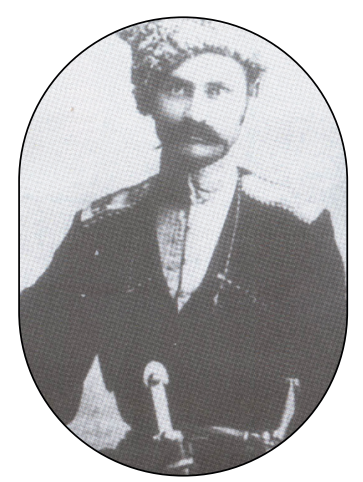

Issu de la dynastie des khans de Crimée. En 1914, le Sultan Gerej fait partie de la Division de cavalerie tcherkesse, composée de musulmans volontaires, la Division sauvage [Dikaja divizija]. En août 1917, il participe en tant que colonel avec Kornilov à son " putsch ", puis commande la Division sauvage au sein de l'armée des volontaires. Il se replie en Crimée, auprès de Wrangel. En exil, il s'active à la création d'une république nord-caucasienne, puis à la formation de troupes caucasiennes auprès du Troisième Reich. À la tête d'une cavalerie cosaque dans le corps d'armée du général Krasnov, puis d'une division caucasienne, il est transféré en Italie au début de 1943, et interné par les Anglais à Oberdrauburg. Le 28 mai 1945, il est livré au NKVD avec 125 officiers caucasiens. Il est jugé à Moscou et pendu.

Sergej Ulagaj étudie à l'école de cavalerie Nicolas, participe à la guerre russo-japonaise, commande la division des cosaques du Kouban pendant la guerre et est décoré de l'Ordre de Saint-Georges.

Partisan de Kornilov, il est arrêté, s'échappe et retourne au Kouban.

Il participe à la première campagne du Kouban, commande la deuxième division du Kouban, libère Stavropol, le Terek, et défait la cavalerie de Dumenko en mars 1919. Il participe à la prise de Tsaritsyne en juin 1919. Sous le commandement de Wrangel, il réussit un débarquement au Kouban les 14-17 août 1920, sans pourtant parvenir à rallier les cosaques. En exil à Marseille, il fonde une troupe de cavaliers voltigeurs en tournées en Europe et en Amérique.

Né à Petropavlovsk en 1884, Ivan Sorokin est le fils d'un riche cosaque. Il devient officier en 1915. Il commande les forces rouges du nord-Caucase, avec l'appui de Staline qui couvre ses excès.

Le 21 octobre 1918, il commet une tentative de coup d’État. Condamné par le Congrès des Soviets à Stavropol, il est fusillé pour trahison le 27 octobre 1918. 
Figure 9. Sergej MARKOV

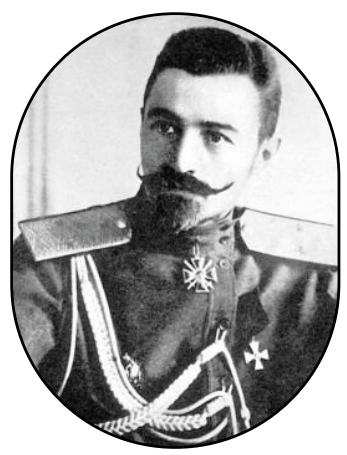

Né d'une famille d'officiers, Sergej Markov étudie à l'académie d'étatmajor en Extrême-Orient, puis participe à la guerre russo-japonaise, en Mandchourie. Entre 1911 et 1914, il enseigne à l'école militaire d'état-major Nicolas. En 1914, il combat dans la « division de fer » de Denikin. Après l'affaire Kornilov, il est enfermé à Bykhov.

En novembre 1917, il devient l'un des fondateurs de l'armée des volontaires. Il participe à la campagne de glace et il est l'auteur d'un fait d'armes légendaire, à savoir la destruction d'un train blindé.

Dans la seconde campagne du Kouban, Markov commande le premier régiment des officiers, mais est grièvement blessé à Chablievka. Il meurt à Metchërtinskaïa le 7 juillet 1918. Son unité devient le régiment, puis la division Markov.

Figure 10. Ivan ERDELY

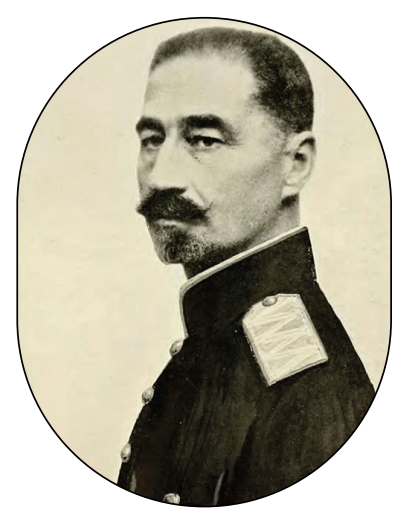

Descendant de comtes hongrois installés en Russie au XVIIIe siècle, Ivan Erdely devient général-major en 1910 et commande le régiment des dragons de la Garde. En 1914, il commande la deuxième division de la cavalerie de la Garde. En 1916, il est lieutenant-général. En juin-juillet 1917, il commande la onzième armée dans l'offensive de Brusilov. Il est arrêté et emprisonné avec Kornilov après le « putsch » d'août. Évadé, il participe à la formation de l'armée des volontaires et à la première campagne du Kouban. Il commande une brigade, puis la première division de la cavalerie des volontaires. En juillet 1919, il devient le commandant en chef des troupes du Caucase du nord. En avril 1920, il est versé dans la réserve des Forces Armées du Sud de la Russie. Exilé en France, en 1937, il est nommé responsable de la commission d'enquête chargée de la trahison de Nikolaj Skoblin. Il décède le 7 juillet 1939 et repose au cimetière russe de Sainte-Geneviève-des-Bois.

\section{Figure 11. Afrikan BOGAEVSKIJ}

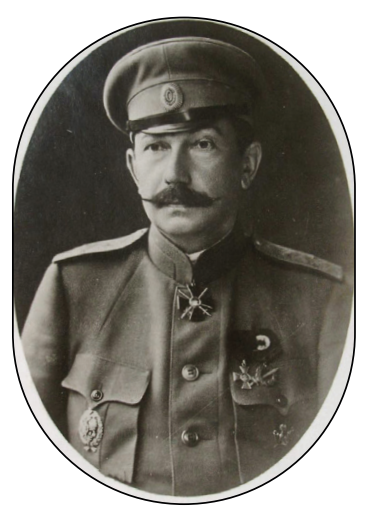

Né d'une famille noble de cosaques du Don, Afrikan Bogaevskij étudie à l'École de cavalerie Nicolas, à l'académie de l'état-major général. En 1914, il commande le quatrième régiment de hussards de Marioupol et, en 1917, la première division de cavalerie de la Garde. En octobre 1917, il gagne Novotcherkass, l'armée du Don sous le commandement de Kaledin, puis participe à la première campagne du Kouban à la tête du régiment des partisans dans l'armée des volontaires. Ensuite, il préside le conseil du gouvernement militaire du Don et rejoint Wrangel en Crimée. Au sein de l'émigration, il œuvre à la création d'une union des combattants cosaques. 
Figure 12. Pëtr WRANGEL

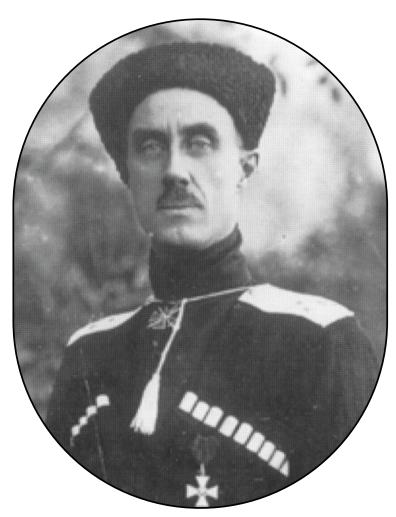

Issu d'une ancienne famille germano-balte, Pëtr Wrangel va d'abord à l’École des Mines à Saint-Pétersbourg, puis à l'École de cavalerie. En 1914, il est capitaine de la Garde à cheval et décoré de l'Ordre de Saint-Georges. En 1916, il devient commandant du premier régiment des cosaques du Transbaïkal et participe à l'offensive de Brusilov. En 1917, il est commandant de la deuxième brigade cosaque de l'Oussouri. Opposé à l'abdication du tsar, il propose l'envoi de troupes à Petrograd. Désavoué, sanctionné, il se retire en Crimée. Déçu par le nationalisme de l'hetman Pavlo Skoropadsky, son ancien camarade d'études, il rejoint Denikin au Kouban, en septembre 1918. À la tête d'une division cosaque, puis de l'armée du Caucase, il conquiert le Kouban, le bassin du Terek, Rostov-sur-le-Don et Tsaritsyne en juin 1919. Chargé durant la marche sur Moscou de prendre Saratov, puis NijniNovgorod avec des forces épuisées, il critique le plan de Denikin qui divise ses troupes. Fin 1919, en désaccord total, il se retire à Istanbul. En mars 1920, Denikin doit démissionner. Le 4 avril, Wrangel reçoit les pleins pouvoirs. Il réorganise l'Armée russe en Crimée, 25000 hommes. Pendant la guerre des bolcheviks contre la Pologne de Josef Pilsudski, il reprend Melitopol et la Tauride du Nord. En septembre, les bolcheviks, quatre fois plus nombreux, reprennent l'offensive contre l’isthme de Perekop. Repoussés le 28 octobre, ils s'en emparent le 7 novembre. Wrangel prépare l'évacuation : 146000 personnes dont 70000 soldats. En exil, Wrangel crée le ROVS, s'installe comme ingénieur à Bruxelles et meurt probablement empoisonné par la Guépéou. Il laisse des Mémoires dont l'unique édition est épuisée.

Open Access Publications - Bibliothèque de l'Université de Genève

Creative Commons Licence 4.0

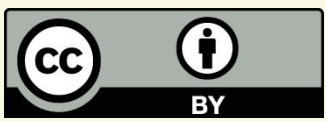

